\title{
Pure and Efficient Single-Photon Sources by Shortening and Functionalizing Air-Suspended Carbon Nanotubes
}

\author{
Rintaro Kawabe, ${ }^{\dagger}$ Hiroshi Takaki,${ }^{\dagger}$ Takayuki Ibi, ${ }^{\dagger}$ Yutaka Maeda,${ }^{\dagger}$ Kenta Nakagawa,,${ }^{\dagger} \S$ and Hideyuki Maki $*, \dagger, \perp$ \\ †Department of Applied Physics and Physico-Informatics, Keio University, Yokohama 223-8522, Japan \\ Department of Chemistry, Tokyo Gakugei University, Koganei, Tokyo 184-8501, Japan \\ ${ }^{\S}$ Kanagawa Institute of Industrial Science and Technology (KISTEC), Ebina 243-0435, Japan \\ ${ }^{\perp}$ Center for Spintronics Research Network, Keio University, Yokohama 223-8522, Japan \\ *Corresponding Author: Hideyuki Maki \\ E-mailmaki@appi.keio.ac.jp
}


Figure S1 shows experimental and simulated PL decay curves of pristine SWCNTs. The details of the measured sample and measurement methods are described in Ref. S1. In the experiment, we used an air-suspended pristine $(9,8)$ SWCNT with a length of $2 \mu \mathrm{m}$ and an emission wavelength of $1298 \mathrm{~nm}$. The excitation power and wavelength were $10 \mu \mathrm{W}$ and $800 \mathrm{~nm}$, respectively. The time resolution was $4 \mathrm{ps}$. Our simulation condition was $L=2 \mu \mathrm{m}, l=300 \mathrm{~nm}$, and $N_{\mathrm{g}}=10$. The value of $l$ for a $(9,8)$ SWCNT under $10 \mu \mathrm{W}$ excitation is $280 \mathrm{~nm}$ was obtained from Ref. S2. For comparison of the experimental result, we calculated the theoretical PL decay by using convolution of the instrumental response function (IRF) of our experimental PL measurement system with the theoretical PL decay curve. The simulated result convoluted with IRF was in good accordance with the experimental result.

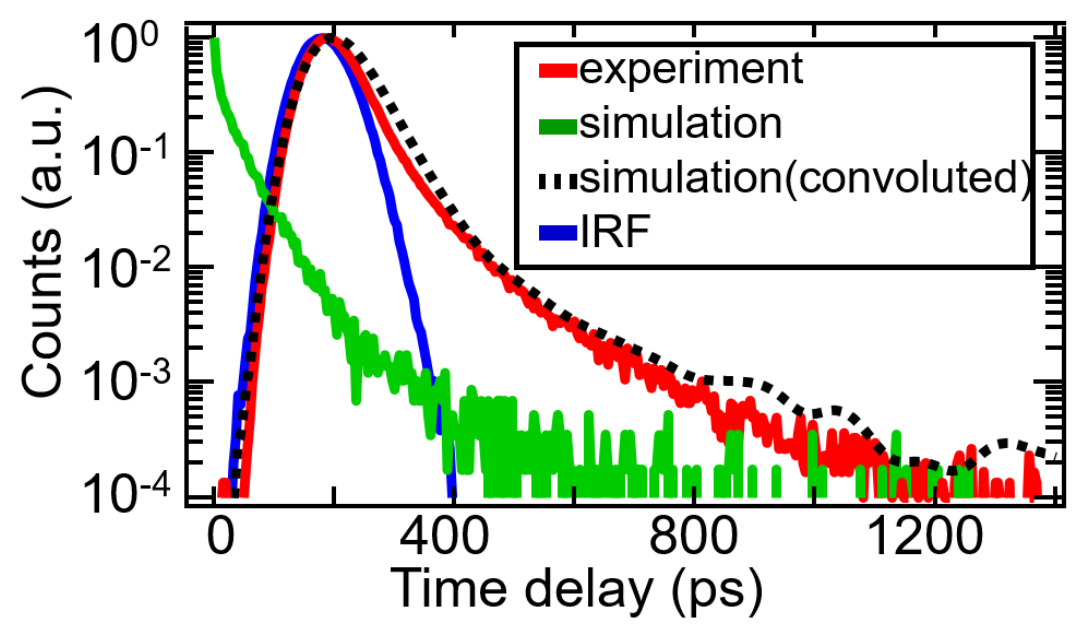

Figure S1. Experimental (red) and simulated (black) PL decays of air-suspend pristine SWCNTs. The simulated PL decay is obtained by using convolution of the instrumental response function (IRF) (blue) of our experimental PL measurement system with the theoretical PL decay curve (green). The intensities of all curve are normalized.

Table S1 shows the constants used in our simulation. The condition of diffusion length $l$, excitation intensity $N_{\mathrm{g}}$, and SWCNTs length $L$ are shown in the main text. A supplementary explanation will be given for our simulation assumptions.

EEA occurs when free excitons meet. End quenching occurs when free excitons reach the ends of the SWCNT. We assumed that one defect was introduced per one functionalized SWCNT. Therefore, it is not necessary to consider the interaction between trapped excitons. When one exciton trapped into the defect, the other exciton is not trapped by the defect because of state filling.

Table S1. List of constants used for our simulation. Each constant corresponds to Figures $1 \mathrm{~b}$ and $1 \mathrm{c}$. Note that $k_{\mathrm{BT}}$ and $k_{\mathrm{DT}}$ in Figure 1c are dominated by exciton diffusion (i.e., when a free exciton moves to the defect position, the exciton falls into the defect unless another exciton is trapped. See the main text.).

\begin{tabular}{|c|c|c|c|c|c|}
\hline & $1 / k_{\mathrm{B}}$ & $1 / k_{\mathrm{D}}$ & $1 / k_{\mathrm{up}}$ & $1 / k_{\mathrm{down}}$ & $1 / k_{\mathrm{T}}$ \\
\hline pristine CNT & $50 \mathrm{ps}$ & $500 \mathrm{ps}$ & $4.295 \mathrm{~ns}$ & $3.536 \mathrm{~ns}$ & - \\
\hline functionalized CNT & $50 \mathrm{ps}$ & $500 \mathrm{ps}$ & $4.295 \mathrm{~ns}$ & $3.536 \mathrm{~ns}$ & $300 \mathrm{ps}$ \\
\hline
\end{tabular}


Figure S2 shows 2D phase diagram plotting $g^{2}(0)$ and $<n>$ as a function of $L / l$ for pristine and functionalized SWCNTs under strong and weak excitation. For pristine SWCNTs, $g^{2}(0)$ is 0.3 or more regardless of the excitation intensity. Furthermore, when the pristine SWCNTs length $L$ is short, the generation efficiency of photons per pulse is very small with no dramatic improvement in $g^{2}(0)$. Hence, it is impossible to realize high purity and high efficiency single-photon sources by using pristine SWCNTs. On the other hand, for functionalized SWCNTs, $g^{2}(0)$ decreases significantly with shortening the length of functionalized SWCNTs, indicating that single-photon purity is remarkably improved. Furthermore, under strong excitation condition $N_{\mathrm{g}}=100$, high efficiency $<n>$ can also be realized. It is important to note that in the case of too short functionalized SWCNTs, the value of $g^{2}(0)$ is smaller, but $<n>$ decrease (see the red square plot with $L / l=0.02$ in Figure S2). In the case of extremely short SWCNTs, it is necessary to make unrealistic strong excitation intensities in order to realize high the single-photon generation efficiency. Therefore, as shown in Figure 8, optimal lengths of SWCNTs and excitation intensities exist in order to realize high single-photon purity and generation efficiency

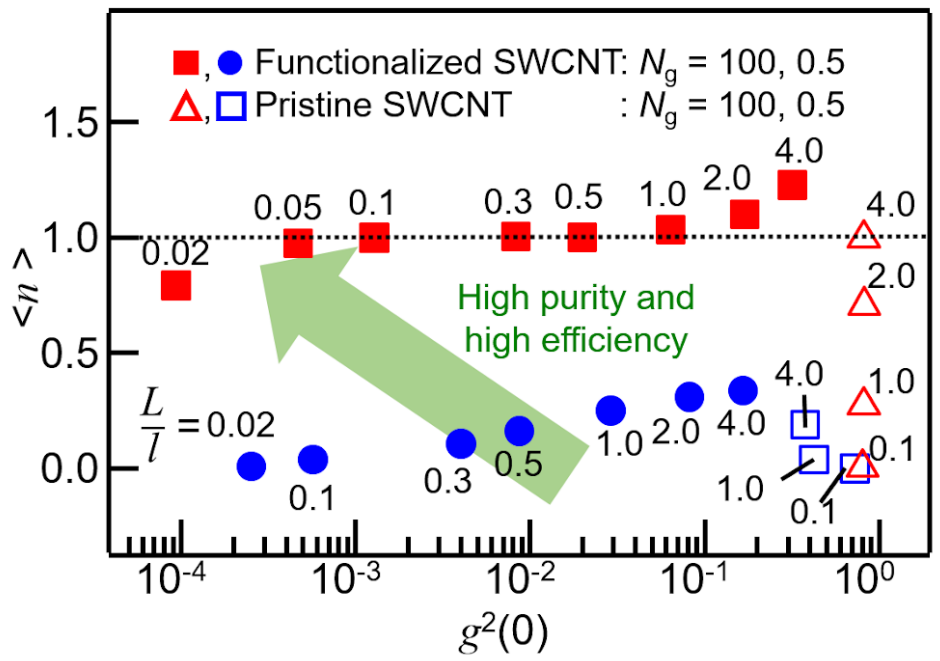

Figure S2. 2D phase diagram of $\left\langle n>\right.$ and $g^{2}(0)$ with various the ratio of diffusion length $l$ to pristine and functionalized SWCNTs length $L$. Functionalized SWCNTs at $N_{\mathrm{g}}=0.5,100$ (red square, blue circle) and pristine SWCNTs at $N_{\mathrm{g}}=0.5$, 100 (open red triangle, open blue square) are shown here. The numerical value in the figure indicates $L / l$. The green arrow indicates that the upper left region has high single-photon purity and high single-photon generation efficiency.

\section{References}

S1. Endo, T.; Ishi-Hayase, J.; Maki, H. Photon Antibunching in Single-Walled Carbon Nanotubes at Telecommunication Wavelengths and Room Temperature. Appl. Phys. Lett. 2015, 106, 113106.

S2. Moritsubo, S.; Murai, T.; Shimada, T.; Murakami, Y.; Chiashi, S.; Maruyama, S.; Kato, Y. K. Exciton Diffusion in Air-Suspended Single-Walled Carbon Nanotubes. Phys. Rev. Lett. 2010, 104, 247402. 